\title{
HOW TO COMPUTE THE SQUARE ROOT OF THE NON-EUCLIDEAN WAVE OPERATOR
}

\author{
M. KOVALYOV AND M. LÉGARÉ
}

(Communicated by J. Marshall Ash)

\begin{abstract}
In this paper we derive a first-order differential operator that can serve as an alternative to the non-Euclidean wave operator to study $\operatorname{Sl}(2, R)$.
\end{abstract}

Extensive research has been done on the harmonic analysis of $\operatorname{SL}(2, R)$. It has been shown [1], [2] that most of the results can be obtained using the theory of the non-Euclidean wave equation and an operator associated with it which behaves roughly like its square root. The question of how to compute a first-order differential operator that can be thought of as the square root of the non-Euclidean wave operator naturally arises. (Obviously, the Dirac operator does not do the job [3].) In this note, we show how it may be done. The operator constructed can be used to provide an analysis of $\operatorname{SL}(2, R)$ different from the approach developed in [1].

But first, let us introduce some notation. Let $\prod$ be the set $\{y \geq 0,-\infty<$ $t, x<+\infty\}$ with the metric form

$$
d \sigma^{2}=d t^{2}-\frac{d x^{2}+d y^{2}}{y^{2}}
$$

The wave operator on $\prod$ is defined to be [1]

$$
\square_{H}=\frac{\partial^{2}}{\partial t^{2}}-y^{2}\left(\frac{\partial^{2}}{\partial x^{2}}+\frac{\partial^{2}}{\partial y^{2}}\right)-\frac{1}{4}
$$

Our objective is to construct a differential operator $M$, such that $M^{2}=\square_{H}$.

In order to do this, we introduce an auxiliary Minkowski space with coordinates $\tau, \chi, \eta$ and the metric form

$$
d s^{2}=d \tau^{2}-d \chi^{2}-d \eta^{2}
$$

Received by the editors January 3, 1989.

1980 Mathematics Subject Classification (1985 Revision). Primary 35P25. 
The transformation

$$
\begin{aligned}
\tau & =\frac{e^{t}}{2}\left(\frac{1}{y}+y+\frac{x^{2}}{y}\right), \\
\chi & =\frac{e^{t}}{2}\left(\frac{1}{y}-y-\frac{x^{2}}{y}\right), \\
\eta & =e^{t} \frac{x}{y}
\end{aligned}
$$

maps $\Pi$ onto the interior of the future light cone $\tau^{2}-\chi^{2}-\eta^{2}>0$ of the Minkowski space in such a way that the metric forms satisfy the identity

$$
d \tau^{2}-d \chi^{2}-d \eta^{2}=e^{2 t}\left(d t^{2}-\frac{d x^{2}+d y^{2}}{y^{2}}\right) .
$$

Let $v(t, x, y)$ be a solution of the hyperbolic wave equation

$$
\square_{H} v=\frac{\partial^{2} v}{\partial t^{2}}-y^{2}\left(\frac{\partial^{2} v}{\partial x^{2}}+\frac{\partial^{2} v}{\partial y^{2}}\right)-\frac{1}{4} v=0 \text {. }
$$

Then the function

$$
u(\tau, \chi, \eta)=e^{-t / 2} v(t, x, y)
$$

is a solution of the Euclidean wave equation

$$
\square_{E} u(\tau, \chi, \eta)=\frac{\partial^{2} u}{\partial \tau^{2}}-\frac{\partial^{2} u}{\partial \chi^{2}}-\frac{\partial^{2} u}{\partial \eta^{2}}=0,
$$

provided $t, x, y$ are expressed in terms of $\tau, \chi, \eta$ via the transformation (2).

One can construct the Dirac operator for $\square_{E}$ as

$$
\mathscr{D}=\sigma^{0} \frac{\partial}{\partial \tau}+\sigma^{1} \frac{\partial}{\partial \chi}+\sigma^{2} \frac{\partial}{\partial \eta},
$$

where

$$
\sigma^{0}=\left(\begin{array}{cc}
0 & -1 \\
1 & 0
\end{array}\right), \quad \sigma^{1}=\left(\begin{array}{ll}
0 & 1 \\
1 & 0
\end{array}\right), \quad \sigma^{2}=\left(\begin{array}{cc}
1 & 0 \\
0 & -1
\end{array}\right) .
$$

Expressing $\mathscr{D}$ in terms of $t, x, y$ we obtain

$$
\begin{aligned}
\mathscr{D}= & \left(\sigma^{0} \frac{\partial t}{\partial \tau}+\sigma^{1} \frac{\partial t}{\partial \chi}+\sigma^{2} \frac{\partial t}{\partial \eta}\right) \frac{\partial}{\partial t} \\
& +\left(\sigma^{0} \frac{\partial x}{\partial \tau}+\sigma^{1} \frac{\partial x}{\partial \chi}+\sigma^{2} \frac{\partial x}{\partial \eta}\right) \frac{\partial}{\partial x} \\
& +\left(\sigma^{0} \frac{\partial y}{\partial \tau}+\sigma^{1} \frac{\partial y}{\partial \chi}+\sigma^{2} \frac{\partial y}{\partial \eta}\right) \frac{\partial}{\partial y} \\
= & e^{-t}\left\{T \frac{\partial}{\partial t}+X \frac{\partial}{\partial x}+Y \frac{\partial}{\partial y}\right\},
\end{aligned}
$$


where

$$
\begin{aligned}
& T=\left(\begin{array}{cc}
-\frac{x}{y} & \frac{y^{2}+x^{2}}{y} \\
-\frac{1}{y} & \frac{x}{y}
\end{array}\right), \\
& X=\left(\begin{array}{cc}
y & -2 x y \\
0 & -y
\end{array}\right), \\
& Y=\left(\begin{array}{cc}
-x & x^{2}-y^{2} \\
-1 & x
\end{array}\right) .
\end{aligned}
$$

In terms of variables $\tau, \chi, \eta, u=\left(u_{1}, u_{2}\right)$, the Dirac operator $\mathscr{D}$ satisfies the equation

$$
\mathscr{D}^{2} u=\square_{E} u=0
$$

Thus, in terms of variables $t, x, y, v=\left(v_{1}, v_{2}\right)$, it must satisfy

$$
\mathscr{D}^{2} e^{t / 2} v=\square_{H} v=0 \text {, }
$$

or

$$
\widetilde{M}^{2} v=\square_{H} v=0
$$

where

$$
\widetilde{M}=e^{t / 2} \mathscr{D} e^{-t / 2}=e^{-t / 2}\left\{T \frac{\partial}{\partial t}+X \frac{\partial}{\partial x}+Y \frac{\partial}{\partial y}\right\} e^{-t / 2} .
$$

Using the fact that $X=-T Y$ and $Y=T X$, we rewrite (11) as

$$
\begin{aligned}
\widetilde{M} & =e^{-t / 2} T\left\{\frac{\partial}{\partial t}-Y \frac{\partial}{\partial x}+X \frac{\partial}{\partial y}\right\} e^{-t / 2} \\
& =e^{-t} T\left\{\frac{\partial}{\partial t}-Y \frac{\partial}{\partial x}+X \frac{\partial}{\partial y}-\frac{1}{2}\right\} .
\end{aligned}
$$

Define

$$
\begin{aligned}
M & =\frac{\partial}{\partial t}-Y \frac{\partial}{\partial x}+X \frac{\partial}{\partial y}-\frac{1}{2} \\
& =\frac{\partial}{\partial t}+\left(\begin{array}{cc}
x & -x^{2}+y^{2} \\
1 & -x
\end{array}\right) \frac{\partial}{\partial x}+\left(\begin{array}{cc}
y & -2 x y \\
0 & -y
\end{array}\right) \frac{\partial}{\partial y}-\frac{1}{2} .
\end{aligned}
$$

One can also compute all eigenfunctions of the time-independent part of (12). Let

$$
L=\left(\begin{array}{cc}
x & -x^{2}+y^{2} \\
1 & -x
\end{array}\right) \frac{\partial}{\partial x}+\left(\begin{array}{cc}
y & -2 x y \\
0 & -y
\end{array}\right) \frac{\partial}{\partial y}-\frac{1}{2} .
$$

Suppose $u(x, y)$ is an eigenfunction of $L$ corresponding to an eigenvalue $\lambda$. Then $e^{-\lambda t} u(x, y)$ is in the kernel of (12), and therefore it is a solution of (4), which implies that $u(x, y)$ is an eigenfunction of the non-Euclidean Laplacian

$$
y^{2}\left(\frac{\partial^{2}}{\partial x^{2}}+\frac{\partial^{2}}{\partial y^{2}}\right)+\frac{1}{4}
$$


corresponding to the eigenvalue $\lambda^{2}$. But according to $[4$, p. 60] any eigenfunction of (14) corresponding to an eigenvalue $\lambda$ can be represented as

$$
f_{\lambda}(x, y) \int_{0}^{2 \pi}\left[\frac{y}{y^{2}+(x-\tan \varphi)^{2}}\right]^{\lambda+1 / 2} d T(\varphi),
$$

where $T(\varphi)$ is a measure on $S^{1}$. The question is elaborated in [4].

Thus we can conclude that any eigenfunction of (13) corresponding to an eigenvalue $\lambda$ can be represented as

$$
f_{\lambda}(x, y)=\int_{0}^{2 \pi}\left[\frac{y}{y^{2}+(x-\tan \varphi)^{2}}\right]^{\lambda+1 / 2}\left(\begin{array}{cc}
\sin & \varphi \\
\cos & \varphi
\end{array}\right) d T(\varphi) .
$$

1. P. Lax and R. Phillips, Scattering theory for automorphic functions, Ann. of Math. Stud. 87 (1976).

2. __ The asymptotic distribution of lattice points in Euclidean and non-Euclidean spaces, J. Funct. Anal. 46 (1982).

3. Y. Choquet-Bruhat, C. De Witt-Morette, and M. Dillard-Bleick, Analysis, manifolds and physics, North-Holland, New York, 1982.

4. S. Helgason, Topics in harmonic analysis on homogeneous spaces, Progress in Math. 13 (1981).

Department of Mathematics, University of Alberta, Edmonton, Alberta T6G 2G1, CANADA 